\title{
Convective instability of autocatalytic reaction fronts in vertical cylinders
}

\author{
Desiderio A. Vasquez \\ Department of Physics, West Virginia University, Morgantown, West Virginia 26506
}

Joseph W. Wilder

Department of Mathematics, West Virginia University, Morgantown, West Virginia 26506

Boyd F. Edwards

Department of Physics, West Virginia University, Morgantown, West Virginia 26506

(Received 18 March 1992; accepted 15 July 1992)

\begin{abstract}
Linear stability analysis predicts that the onset of convection for an ascending autocatalytic reaction front in a vertical cylinder corresponds to a nonaxisymmetric mode. This mode consists of a single convective roll confined to the region near the reaction front, with fluid rising in half of the cylinder and falling in the other half. Experiments show a flat front below the onset of convection and an axisymmetric front well above the onset of convection. New experiments are called for to closely examine the onset of convection in order to test this prediction.
\end{abstract}

\section{INTRODUCTION}

Hydrodynamic stability theory is needed to understand recent experimental observations of convection near autocatalytic reaction fronts. These experiments ${ }^{1,2}$ on ascending iodate-arsenous acid reaction fronts ${ }^{3-5}$ in vertical capillary tubes show that steady axisymmetric convection can exist in the frame of the moving front. This convection is driven by the buoyancy of the lighter reacted fluid below the ascending front; descending fronts involve no convection. The goal of this paper is to present the results of a complete linear hydrodynamic stability analysis including both axisymmetric and nonaxisymmetric modes. As will be seen, this analysis predicts a transition to nonaxisymmetric convection that should be observable in experiments.

The bulk of the previous work on autocatalytic systems emphasizes reaction-diffusion mechanisms ${ }^{6}$ for front propagation rather than hydrodynamic stability. In these systems, a reaction front, or "chemical wave," is limited in propagation speed by the slow molecular diffusion of a catalyst into the unreacted fluid mixture. Since the "autocatalytic" reaction produces its own catalyst, the reaction front can propagate indefinitely. Reaction-diffusion mechanisms account for spiral waves, ${ }^{7}$ chemical chaos, ${ }^{8}$ and Turing patterns ${ }^{9}$ in autocatalytic reactions such as the Belousov-Zhabotinski reaction, but cannot account for convection. Such convection is driven by density gradients produced by thermal and chemical gradients in the vicinity of the reaction front. In fluid problems such as the Rayleigh-Bénard problem of a fluid heated from below, such buoyancy-driven convection can take the form of traveling waves, ${ }^{10}$ oscillatory instabilities, period doubling, mode locking, and convective chaos. ${ }^{11}$ Involving both hydrodynamic and reaction-diffusion effects, convection in autocatalytic systems is a potentially rich and fruitful area of investigation.

A crucial step toward understanding convection in autocatalytic systems is to understand the onset of convection. Previous work by Pojman and Epstein ${ }^{4}$ made use of calculations by Taylor ${ }^{12}$ and Wooding ${ }^{13}$ for onset of free convection to estimate the critical parameters for the onset of convection in autocatalytic systems. Free convection is driven by a vertical concentration gradient and an associated density gradient both of which are uniform over the entire length of a long vertical cylinder; free convection does not involve a reaction front. The corresponding flow is in the vertical direction only and occurs over the entire length of the cylinder, with uplow in one half of the cylinder and downflow in the opposing half. In contrast, autocatalytic systems involve density gradients only in the vicinity of a propagating chemical reaction front, which lead to more complicated two- and three-dimensional flows restricted to the neighborhood of the front. Thus, in autocatalytic systems, the density changes with time as the front propagates, which is not true for free convection.

A theory of convection near autocatalytic reaction fronts ${ }^{14}$ treats the thin chemical reaction front as a moving surface that consumes unreacted fluid of mass density $\rho_{u}$ to produce reacted fluid of lower density $\rho_{r}$ thereby relegating all chemical reactions to the surface and precluding a molecular diffusion equation. The theory is relevant to reactions such as the iodate-arsenous acid reaction, which allows only a single front passage through the fluid. The corresponding dimensionless driving parameter appropriate for a long vertical cylinder and for uniform $\rho_{r}$ and $\rho_{u}$ (Ref. 15),

$$
S=\delta g a^{3} / v D_{C},
$$

involves a fractional density difference $\delta=\left(\rho_{u}-\rho_{r}\right) / \rho_{r}$ the acceleration of gravity $g$, the cylinder radius $a$, the kinematic viscosity $v$, and the molecular diffusivity $D_{C}$ of the catalyst. The small chemical concentrations typical of experiments imply small $\delta \approx 10^{-4}$ and negligible differences in $v$ and $D_{C}$ between the reacted and unreacted fluids. The driving parameter $S$ measures the strength of buoyancy, which tends to destabilize a flat front in favor of convection, relative to curvature effects, which tend to flatten the front. In the linear stability theory, convection occurs above a critical value $S_{c}$, where curvature effects and buoy- 
ancy exactly balance each other and the system is marginally stable. Although the experimental observations of axisymmetric convection well above the onset of convection prompted a calculation of $S_{c}=370.2$ for onset of axisymmetric convection, ${ }^{15}$ a complete stability analysis also requires nonaxisymmetric modes. The purpose of this paper is to perform such a stability analysis, which yields $S_{c}=87.9$ for a nonaxisymmetric mode consisting of a single convective roll confined to the vicinity of the reaction front. The theory therefore predicts this lowest mode near the onset of convection; experiments intended to precisely locate the onset of convection could readily test this prediction. Calculated values of $S_{c}$ are universal, being independent of the cylinder radius and any fluid parameters, and therefore apply generally to experiments.

Despite the typically exothermic reaction, the density can be considered as piecewise uniform in two important limits. ${ }^{14}$ Infinite thermal diffusivity renders the temperature uniform everywhere with $\delta=\delta_{1}>0$ measuring the fractional density difference between the fluids due to their different chemical compositions. Zero thermal diffusivity precludes heat flow and renders the temperature piecewise uniform, so that the corresponding fractional density difference $\delta=\delta_{0}>\delta_{1}$ also includes the density difference due to thermal expansion. In both limits, a thermal diffusion equation is unnecessary. These limits provide approximate bounds on results for finite thermal diffusivity. Previous arguments ${ }^{15}$ indicate that the thermal diffusivity can be considered as large when the thermal length scale is large compared with the convective length scale; the ratio of these length scales is about 5 in the experiments. Recent calculations for finite thermal diffusivity in a laterally unbounded system ${ }^{16}$ support these arguments.

\section{MATHEMATICAL FORMULATION}

The fluid velocity $\mathbf{v}(\mathbf{x}, t)$ and the reaction front height $z=h(x, y, t)$ evolve according to the dynamical equations $\mathrm{s}^{14}$

$$
\begin{aligned}
& \frac{\partial \mathrm{v}}{\partial t}+(\mathbf{v} \cdot \nabla) \mathbf{v}=-\rho \hat{z}-\nabla P+\nabla^{2} \mathbf{v}, \\
& \nabla \cdot \mathbf{v}=0, \\
& \hat{n} \cdot\left(\hat{z} \frac{\partial h}{\partial t}-\left.\mathbf{v}\right|_{z=h}\right)=v_{0}+\frac{D_{C}}{v} \kappa,
\end{aligned}
$$

where length, time, pressure, and density are measured in units of $a, a^{2} / v, v^{2} \rho_{r} / a^{2}$, and $\tilde{\rho}=v^{2} \rho_{\sqrt{ }} / g a^{3}$, and where we have neglected the small density difference between the reacted and unreacted fluids by setting $\rho=\rho_{r} / \widetilde{\rho}$ everywhere except in the large gravity term [the first term on the right side of Eq. (2a) ], consistent with the Oberbeck-Boussinesq approximation. ${ }^{17}$ The first two equations express force balance and mass continuity. The third relates the normal component of the front velocity relative to the fluid (the left side) to the volume of unreacted fluid consumed per unit front surface area per unit time (the right side) involving a dimensionless consumption rate $v_{0}$ for a flat interface and a correction proportional to the front curvature $\kappa$, measured as positive when the center of curvature is in the unreacted fluid. This curvature correction tends to lower peaks and to raise valleys in the front surface, thus, tending to flatten the front. Again, neglecting the density difference, the matching and boundary conditions include continuous fluid velocity $\mathbf{v}$ and continuous stress $n_{j} T_{i j}$ at the front as well as vanishing velocity at the no-slip impenetrable side walls. Here, the $n_{j}$ are the Cartesian components of the normal vector $\hat{n}$ pointing into the unreacted fluid and $T_{i j}=P \delta_{i j}-\partial v_{i} / \partial x_{j}-\partial v_{j} / \partial x_{i}$ is the dimensionless stress tensor.

To study the onset of convection, it is convenient to introduce a reduced pressure $p=P+\rho z$ and to allow small perturbations about an ascending convectionless flat front described in the comoving frame by $\mathbf{v}^{(0)}=-v_{0} \hat{z}, h^{(0)}=0$, $\hat{n}^{(0)}=\widehat{z}$, and $\kappa^{(0)}=0$. Linearizing in the perturbations yields

$$
\begin{aligned}
& \frac{\partial}{\partial t} \mathbf{v}^{(1)}-v_{0} \frac{\partial}{\partial z} \mathbf{v}^{(1)}=-\nabla p^{(1)}+\nabla^{2} \mathbf{v}^{(1)} \quad(z \neq 0), \\
& \nabla \cdot v^{(1)}=0, \\
& \frac{\partial}{\partial t} h^{(1)}=\frac{D_{C}}{v} \nabla^{2} h^{(1)}+\left.\hat{z}^{\bullet} \mathbf{v}^{(1)}\right|_{z=0},
\end{aligned}
$$

where $\hat{n}^{(1)}=-\nabla h^{(1)}$ and $\kappa^{(1)}=\nabla^{2} h^{(1)}$. Continuous fluid velocity $\mathbf{v}$, tangential stress $\epsilon_{i j k} n_{j} n_{l} T_{k l}$, and normal stress $n_{i} n_{j} T_{i j}$ at the front and the continuity equation require

$$
\begin{aligned}
& {\left[\mathbf{v}^{(1)}\right]_{-}^{+}=0,} \\
& {\left[\frac{\partial}{\partial z} \mathbf{v}^{(1)}\right]_{-}^{+}=0,} \\
& {\left[p^{(1)}\right]_{-}^{+}=\left(D_{C}(v) S h^{(1)}\right.}
\end{aligned}
$$

to first order in the perturbations. Here, $[q]_{-}^{+}$ $=\lim \left(\left.q\right|_{z=+\epsilon}-\left.q\right|_{z=-\epsilon}\right)$ is the difference between the vales of a quantity $q$ above and below the front, which is located at $z=0$ for terms already first order in the perturbations.

We can now specialize to cylindrical coordinates $\mathbf{x}$ $=(r, \theta, z)$ relevant to the experiments. At the onset of convection where $S=S_{c}$, the perturbations neither grow nor decay with time so that $\partial / \partial t=0$. Accordingly, we can use general velocity perturbations

$$
\mathbf{v}_{k}^{(1)}=\left[u_{k}(r) \cos m \theta, v_{k}(r) \sin m \theta, w_{k}(r) \cos m \theta\right] e^{k z}
$$

with integer $m$ to rewrite Eqs. (3a) and (3b) as

$$
\begin{aligned}
& k\left(\nabla^{2} u_{k}-2 m \frac{v_{k}}{r^{2}}-\frac{u_{k}}{r^{2}}\right)-\frac{\partial}{\partial r} \nabla^{2} w_{k}=0, \\
& k\left[r \nabla^{2} v_{k}-2 m\left(u_{k} / r\right)-v_{k} / r\right]+m \nabla^{2} w_{k}=0, \\
& \frac{1}{r} \frac{\partial}{\partial r}\left(r u_{k}\right)+m \frac{v_{k}}{r}+k w_{k}=0,
\end{aligned}
$$

with

$$
\nabla^{2}=\frac{1}{r} \frac{\partial}{\partial r} r \frac{\partial}{\partial r}-\frac{m^{2}}{r^{2}}+k^{2}
$$


Equations (5a) and (5b) were obtained from the vector relation ( $3 a$ ) by eliminating the pressure. The second term in Eq. (3a) was neglected because it leads to corrections in $S_{c}$ of relative order $10^{-5}$ (Ref. 7). For fixed $m$, Eqs. (5) are a system of eigenvalue equations for $k$. Scparation of variables ${ }^{18}$ is not applicable because of the rigid boundary conditions, hence we use the Frobenius method to solve the equations. We assume a power series for each variable:

$$
\begin{aligned}
& u_{k}=r^{a} \sum_{n=0}^{\infty} a_{n} r^{n}, \\
& v_{k}=r^{b} \sum_{n=0}^{\infty} b_{n} r^{n},
\end{aligned}
$$

and

$$
w_{k}=r^{r} \sum_{n=0}^{\infty} c_{n} r^{n}
$$

To obtain values of $a, b$, and $c$ we substitute the leading term of each series $\left(r^{a} a_{0}, r^{b} b_{0}\right.$ and $r^{c} c_{0}$, respectively) into Eqs. (5); this leads to a linear system of equations on $a_{0}$, $b_{0}$, and $c_{0}$. Requiring that the determinant vanishes yields $c=m, a=b=m-1$, and $a_{0}=-b_{0}$. The only free parameters are $c_{0}, b_{0}$, and the coefficient of the second-order term $b_{2}$. Later, these parameters will help us to fix the boundary conditions at the wall. The other second-order coefficients are given by

$$
a_{2}=-\left(k c_{0}+m b_{2}\right) /(m+2)
$$

and

$$
\begin{aligned}
c_{2}= & -\left[k \left(k^{2} m b_{0}+2 k^{2} b_{0}+k m^{2} c_{0}+4 k m c_{0}+4 m^{2} b_{2}\right.\right. \\
& \left.\left.+4 m b_{2}\right)\right] /\left[4 m\left(m^{2}+3 m+2\right)\right],
\end{aligned}
$$

and the remaining terms follow from the recursion relations:

$$
\begin{aligned}
b_{2 n}= & -\left[k \left(k m^{2} a_{2 n-2}+k m^{2} b_{2 n-2}+2 k m n a_{2 n-2}+4 k m n b_{2 n-2}-2 k m b_{2 n-2}+4 k n^{2} b_{2 n-2}-4 k n b_{2 n-2}-4 m^{2} n c_{2 n-2}\right.\right. \\
& \left.\left.+4 m^{2} c_{2 n-2}-4 m n^{2} c_{2 n-2}+8 m n c_{2 n-2}-4 m c_{2 n-2}\right)\right] /\left[16 n\left(m^{2} n-m^{2}+2 m n^{2}-3 m n+m+n^{3}-2 n^{2}+n\right)\right], \\
a_{2 n}= & -\left(k^{2} m a_{2 n-2}+k^{2} m b_{2 n-2}+2 k^{2} n b_{2 n-2}-2 k^{2} b_{2 n-2}+4 m^{2} n b_{2 n}-4 m^{2} b_{2 n}+12 m n^{2} h_{2 n}-16 m n b_{2 n}+4 m b_{2 n}+8 n^{3} b_{2 n}\right. \\
& \left.-16 n^{2} b_{2 n}+8 n b_{2 n}\right) /\left[4 m\left(m n-m+n^{2}-2 n+1\right)\right],
\end{aligned}
$$

and

$$
\begin{aligned}
c_{2 n}= & -\left[k \left(2 k^{2} m n b_{2 n-2}+k^{2} m a_{2 n-2}-k^{2} m b_{2 n-2}+2 k^{2} n^{2} b_{2 n-2}-2 k^{2} n b_{2 n-2}+2 k m^{2} n c_{2 n-2}-2 k m^{2} c_{2 n-2}+2 k m n^{2} c_{2 n-2}\right.\right. \\
& -4 k m n c_{2 n-2}+2 k m c_{2 n-2}+8 m^{2} n^{2} b_{2 n}-8 m^{2} n b_{2 n}+16 m n^{3} b_{2 n}-24 m n^{2} b_{2 n}+8 m n b_{2 n}+8 n^{4} b_{2 n}-16 n^{3} b_{2 n} \\
& \left.\left.+8 n^{2} b_{2 n}\right)\right] /\left[8 m n\left(m^{2} n-m^{2}+2 m n^{2}-3 m n+m+n^{3}-2 n^{2}+n\right)\right]
\end{aligned}
$$

for the coefficients of even order. The odd-order coefficients vanish. It is found that including the first 20 nonzero terms in the sums in Eqs. (7) gives excellent convergence.

We find the eigenvalue $k$ from the boundary conditions $u_{k}=v_{k}=w_{k}=0$ in the following way: Since the equations are linear, the eigenfunction is a superposition of three basic solutions obtained from the following three choices for the free parameters:

$$
\left(\begin{array}{l}
b_{0} \\
b_{2} \\
c_{0}
\end{array}\right)=\left(\begin{array}{l}
1 \\
0 \\
0
\end{array}\right),\left(\begin{array}{l}
0 \\
1 \\
0
\end{array}\right) \text { and }\left(\begin{array}{l}
0 \\
0 \\
1
\end{array}\right) .
$$

The velocity vector at $r=1$ is a linear combination of the basic solutions at $r=1$. The vanishing velocity vector at the walls requires that the determinant associated with the linear system at $r=1$ vanishes. This results in an equation for $k$ that can be solved using standard numerical techniques. The eigenfunctions are obtained once the eigenvalue for the system at $r=1$ is known. In practice, this method is useful to find eigenvalues $k$ of small magnitude. For larger magnitudes of $k$, the coefficients in the sums in Eqs. (7) are comparable in magnitude but alternate in sign, creating cancellation problems for $r \rightarrow 1$. Accordingly, for large $k$, the series method is used only up to $r=0.1$, beyond which Eqs. (5) are integrated directly. Direct numerical integration cannot be applied at $r=0$ since the equations are singular at that point. Values of $k$ thus obtained for axisymmetric $(m=0)$ modes agree with our previous axisymmetric results obtained using a Galerkin method. ${ }^{15}$

To determine $S_{c}$, we use the lowest $N$ eigenvalues $k$ with negative real parts to construct a general solution $\mathbf{v}^{(1)}=\Sigma_{i=1}^{N} A_{i} v_{k_{i}}^{(1)}$ for $z>0$ as a linear combination of the $\mathbf{v}_{k}^{(1)}$, and construct a similar solution for $z<0$ using eigenvalues $k$ with positive real parts. Substituting these series into the matching conditions (3d), (3e), and $\left[\partial^{3} w^{(1)} / \partial z^{3}\right]_{-}^{+}=\left.S_{c} w^{(1)}\right|_{z=0} \quad$ [from Eqs. (3a) $-(3 \mathrm{c})$ and (3f)] leads to a homogeneous linear algebraic system for the coefficients. For fixed $m$, the critical values $S_{c}$ for which the determinant vanishes are designated as $S_{m 1}, S_{m 2}$, $S_{m 3}, \ldots$, in ascending order. The number of significant figures in the values of $S_{c}$ quoted below for $N=90$ is based on the convergence for increasing $N$.

\section{RESULTS AND DISCUSSION}

Calculated values of $S_{c}$ and corresponding mode diagrams are shown in Fig. 1. The lowest value $S_{11}=87.9$ 


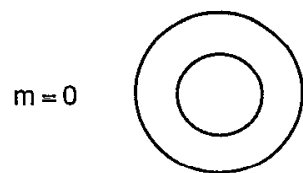

$S_{01}=370.2$

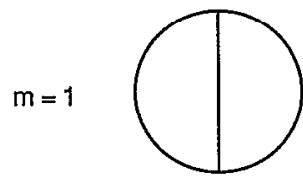

$S_{11}=87.9$

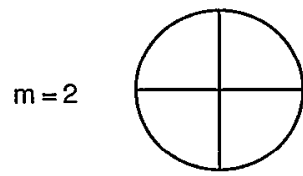

$\mathrm{S}_{21}=297.1$

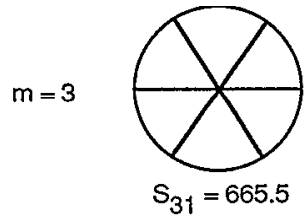

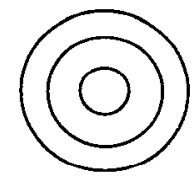

$S_{02}=1835.0$

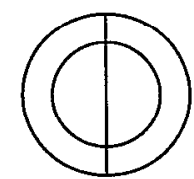

$\mathrm{S}_{12}=884.2$

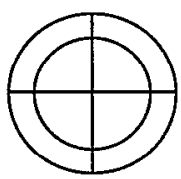

$S_{22}=1668.9$

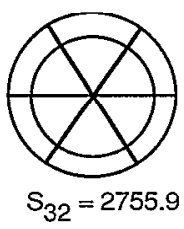

FIG. 1. Calculated critical driving parameters $S_{c}=S_{m n}$ and corresponding mode diagrams for onset of convection near ascending fronts in a vertical cylinder. Solid traces represent nodal surfaces separating regions of rising and falling flow, with the outer circle representing the cylinder itself. The lowest critical value $S_{11}$ corresponds to a nonaxisymmetric mode that dominates at the onsct of convcction.

corresponds to a nonaxisymmetric mode consisting of a single convective roll confined to the vicinity of the reaction front, with regions of rising and falling fluid separated by a vertical nodal plane through the cylinder axis (the $y-z$ plane). A plot of the corresponding perturbation velocities $v^{(1)}$ as seen in a vertical plane perpendicular to the nodal plane (the $x-z$ plane, Fig. 2) shows an exponential decay in the velocity magnitude with vertical distance from the front, indicating that fluid motion is localized essentially to a vertical distance equal to the cylinder diameter, and that the fluid is undisturbed well above and well below the front. This predicted nonaxisymmetric mode for onset of convection is reminiscent of onset modes for Rayleigh-Taylor ${ }^{19}$ and Rayleigh-Bénard ${ }^{20}$ convection in long vertical cylinders.

It is instructive to compare the theory with experiments on iodate-arsenous acid mixtures. In these experiments, flat descending fronts (with the lighter reacted fluid above the front) are stable and absent of convection for all tube radii $a$ studied. Flat ascending fronts propagate at the same speed as descending fronts for $a=0.47 \mathrm{~mm}$ (Fig. 3), whereas for $a=0.89$ and $1.20 \mathrm{~mm}$, ascending fronts propagate at significantly higher speeds and are concave down, axisymmetric, and stationary in the frame moving with the front, indicating the presence of well-developed steady axisymmetric convection in the comoving frame. Evidently, an experimental transition to convection occurs between $a=0.47$ and $0.89 \mathrm{~mm}$. The theory predicts a corresponding critical radius $a_{c}$ for onset of convection through the rela-

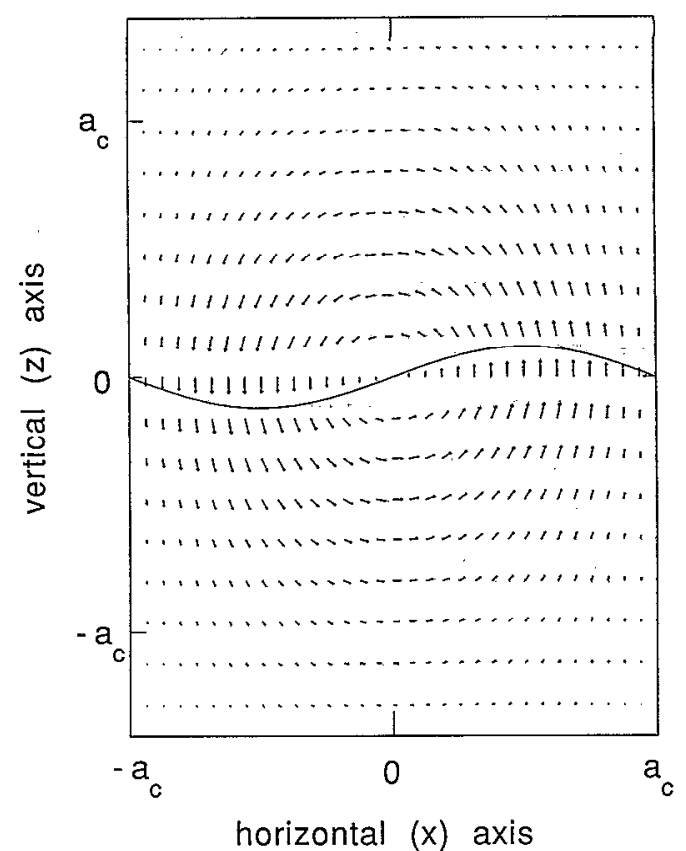

FIG. 2. Velocity field for the predicted lowest nonaxisymmetric mode $\left(S_{11}=87.9\right)$ at the onset of convection in a cross section through the axis of a vertical cylinder that is perpendicular to the nodal plane. The ascending reaction front (represented by the solid trace) consumes unreacted fluid above the front to produce lighter reacted fluid below the front.

tion $S_{c}=\delta g a_{c}^{3} / v D_{C}$ [from Eq. (1)]. The calculated value $S_{c}=S_{1 \mathrm{I}}=87.9$ and the measured values ${ }^{15} \delta_{1}=0.90 \times 10^{-4}$, $\delta_{0}=1.87 \times 10^{-4}, g=980 \mathrm{~cm} / \mathrm{sec}^{2}, v-9.2 \times 10^{-3} \mathrm{~cm}^{2} / \mathrm{sec}$, and $D_{C}=2.0 \times 10^{-5} \mathrm{~cm}^{2} / \mathrm{sec}$ yield the predicted values $a_{c}=0.45$ and $0.57 \mathrm{~mm}$ (closed arrowheads in Fig. 3) for onset of nonaxisymmetric convection for zero $\left(\delta=\delta_{0}\right)$ and infinite $\left(\delta=\delta_{1}\right)$ thermal diffusivity, respectively. We also

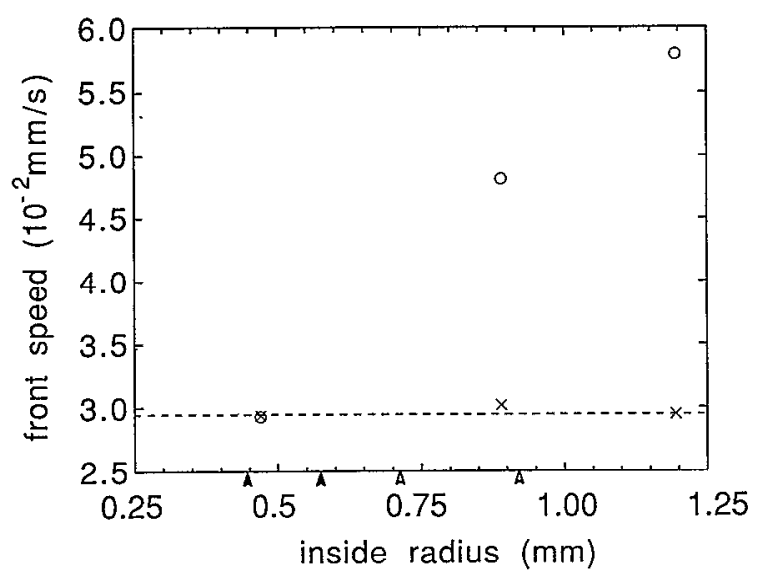

FIG. 3. Experimental ascending $(O)$ and descending $(X)$ front propagation speeds as a function of tube radius $a$ for an iodate-arsenous acid reaction. ${ }^{2}$ The ascending speed deviates from the descending speed $c_{0}=2.95 \pm 0.04 \times 10^{-2} \mathrm{~mm} / \mathrm{sec}$ (dashed line) at and above $a=0.89 \mathrm{~mm}$ where axisymmetric convection is first observed. The closed arrowheads give the predicted critical radii $a_{c}$ for onset of nonaxisymmetric convection with critical driving parameter $S_{11}=87.9$ for zero and infinite thermal diffusivity. The open arrowheads give the predicted critical radii for axisymmetric convection with $S_{01}=370.2$. 
note the corresponding predicted critical radii $a_{c}=0.72$ and $0.92 \mathrm{~mm}$ (Ref. 15, open arrowheads in Fig. 3) for onset of axisymmetric convection with $S_{01}=370.2$, involving upward flow near the center of the cylinder and downward flow near the sides. The theoretical thresholds for both nonaxisymmetric and axisymmetric convection are consistent with the experimental threshold radius between 0.47 and $0.89 \mathrm{~mm}$.

The theory predicts nonaxisymmetric convection near the onset of convection, whereas experiments to date observe only well-developed axisymmetric convection. Clearly, experimental observations intended to precisely locate the onset of convection would be very useful. These could be made either by using the cylinder radius as a control parameter as in the previous experiments, or by using the density difference $\delta$ as a control parameter by varying the reactant concentrations. Calculations for finite thermal diffusivity are not expected to change the symmetry of the lowest unstable mode.

Interesting nonlinear aspects of convection in autocatalytic systems awaiting further investigation include convective enhancements of the front propagation speed; instabilities of the finite-amplitude flow, and the transition to chaos. These investigations may also help to resolve the experimental issues discussed above.

\section{ACKNOWLEDGMENTS}

Discussions with Kenneth Showalter and support from National Science Foundation Grant No. RII-8922106 and the West Virginia University Energy and Water Research Center are gratefully acknowledged.

IT. McManus, "Chemical waves in the indate-arsenous acid reaction," Ph.D. thesis, West Virginia University, Chap. 3, 1989.

2J. A. Pojman, I. R. Epstein, T. J. McManus, and K. Showalter, "Convective effects on chemical waves. 2 . Simple convection in the iodatearsenous acid system," J. Phys. Chem. 95, 1299 (1991).

${ }^{3}$ N. Ganapathisubramanian and K. Showalter, "Relaxation behavior in a bistable chemical system near the critical point and hysterisis limit," $J$. Chem. Phys. 84, 5427 (1986).

${ }^{4}$ J. A. Pojman and I. R. Epstein, "Convective effects on chemical waves. 1. Mechanisms and stability criteria," J. Phys. Chem. 94, 4966 (1990);
J. A. Pojman, I. P. Nagy, and I. R. Epstein, "Convective effects on chemical waves. 3 . Multicomponent convection in the iron(II)-nitric acid system," J. Phys. Chem. 95, 1306 (1991).

${ }^{5} \mathrm{G}$. Bazsa and I. R. Epstein, "Traveling waves in the nitric acid-iron(II) reaction," J. Phys. Chem. 89, 3050 (1985); I. Nagypál, G. Bazsa, and I. R. Epstein, "Gravity-induced anisotropies in chemical waves," J. Am. Chem. Soc. 108, 3635 (1986).

${ }^{6} \mathrm{R}$. J. Field and M. Burger, Oscillations and Traveling Waves in Chemical Systems (Wiley, New York, 1985); A. T. Winfree, When Time Breaks Down (Princeton U.P., Princeton, NJ, 1987).

${ }^{7} \mathrm{~A}$. Karma, "Scaling regime of spiral wave propagation in singlediffusive media," Phys. Rev. Lett. 66, 2274 (1991); A. T. Winfree, Science 175, 634 (1972).

${ }^{8}$ W. T. Tam, J. A. Vastano, H. L., Swinney, and W. Horsthemke, "Regular and chaotic chemical spatiotemporal patterns," Phys. Rev. Lett. 61, 2163 (1988).

${ }^{9}$ I. Lengyel and I. R. Epstein, "Modeling of Turing structures in the chlorite-iodide-malonic acid-starch reaction system," Science 251, 650 (1991).

${ }^{10} \mathrm{P}$. Kolodner, "Drifting pulses of traveling-wave convection," Phys. Rev. Lett. 66, 1165 (1991).

${ }^{11}$ P. Manneville, Dlssipative Structures and Weak Turbulence (Academic, New York, 1990), and references therein.

${ }^{12}$ G. I. Taylor, "Diffusion and mass transport in tubes," Proc. R. Soc. London Ser. B 67, 857 (1954).

${ }^{13} \mathrm{R}$. A. Wooding, "The stability of a viscous liquid in a vertical tube containing porus material," Proc. R. Soc. London Ser. A 252, 120 (1959).

${ }^{14}$ B. F. Edwards, J. W. Wilder, and K. Showalter, "Onset of convection for autocatalytic reaction fronts: Laterally unbounded systems," Phys. Rev. A 43, 749 (1991).

${ }^{15}$ D. A. Vasquez, B. F. Edwards, and J. W. Wilder, "Onset of convection for autocatalytic reaction fronts: Laterally bounded systems," Phys. Rev. A 43, 6694 (1991).

${ }^{16}$ J. W. Wilder, B. F. Edwards, and D. A. Vasquez, "Finite thermal diffusivity at onset of convection in autocatalytic systems: Continuous fiuid density," Phys. Rev. A 45, 2320 (1992).

${ }^{17}$ J. Boussinesq, Theorie Analytique de la Chaleur (Gauthier-Villars, Paris, 1903) Vol. 2; A. Oberbeck, "Uber die Wärmeleitung der Flussigkeiten bei der Berüchsichtingung der Strömungen infolge von Temperaturdifferenzen," Ann. Phys. Chem. (Leipzig) 7, 271 (1879); A. Oberbeck, "Uber die Bewegungsercheinungen der Atmosphere," Sitz. Ber. K. Preuss. Akad. Miss., 383 and 1129 (1888).

${ }^{18} \mathrm{~S}$. Rosenblat, "Thermal convection in a vertical circular cylinder," $\mathrm{J}$. Fluid Mech. 122, 395 (1982).

${ }^{19}$ J. C. Maxwell, Scientific Papers (Cambridge U.P., Cambridge, England, 1890); P. G. Drazin and W. H. Rcid, Hydrodynamic Stability (Cambridge U.P., Cambridge, England, 1981).

${ }^{20} \mathrm{G}$. Z. Gershuni and E. M. Zhukhovitskii, Convective Stability of Incompressible Fluids (Keterpress, Jerusalem, 1976). 